\title{
SOME DIMENSION 3 CASES OF THE CANONICAL ELEMENT CONJECTURE
}

\author{
CRAIG HUNEKE ${ }^{1}$ AND JEE KOH
}

\begin{abstract}
The Canonical Element Conjecture holds for those 3-dimensional local rings of depth 2 whose second local cohomology module is a finite direct sum of cyclic modules, and for those 3-dimensional local rings whose second local cohomology module is cyclic.
\end{abstract}

In this note all rings are assumed to be commutative Noetherian with identity. By $H_{m}^{i}(R)$ we denote the $i$ th local cohomology module of a local ring $(R, m)$ with respect to the maximal ideal $m$. We will prove the following two theorems.

THEOREM 1.1. Let $(R, m)$ be a 3-dimensional local ring of depth 2. Suppose $H_{m}^{2}(R)$ is a finite direct sum of cyclic $R$-modules. Then the Canonical Element Conjecture holds for $R$.

THEOREM 1.2. Let $(R, m)$ be a 3-dimensional local ring. Suppose that $H_{m}^{2}(R)$ is cyclic. Then the Canonical Element Conjecture holds for $R$.

We refer the reader to [6] for a full discussion of the Canonical Element Conjecture, which is due to M. Hochster. We note that in [6] it was shown that this conjecture implies most of the homological conjectures which are known to follow from the existence of big Cohen-Macaulay modules.

Although the Canonical Element Conjecture can be reduced to the case of normal domains [6, (2.4) Corollary] so that the depth condition in Theorem 1 may be assumed in studying the conjecture, this reduction will in general change the local cohomology. If $H_{m}^{2}(R)$ is cyclic, then we can reduce to the depth 2 case to relax the condition on depth but the argument given to prove Theorem 2 does not seem to work when $H_{m}^{2}(R)$ is a direct sum of cyclic modules. We also note that Theorem 1 includes all known examples of 3-dimensional normal domains which are not Cohen-Macaulay purely for "mixed characteristic" reasons. (These are described in [7].) The proof of the Canonical Element Conjecture for these normal domains was first done by P. Roberts by explicit computation.

In our proof we will use an equivalent form of the Canonical Element Conjecture due to Paul Roberts [8]. For the sake of completeness we have included an appendix with the proof of the equivalence, and would like to thank Paul Roberts for

Received by the editors October 26, 1984 and, in revised form, October 1, 1985.

1980 Mathematics Subject Classification (1985 Revision). Primary 13D25, 13H10.

Key words and phrases. Canonical Element Conjecture, local cohomology.

${ }^{1}$ Partially supported by the NSF. 
permitting us to do so. The proof we give is due to the referee of this paper and is shorter than the original proof given in [8]. If $\mathbf{x}$ is the sequence of elements $x_{1}, \ldots, x_{n}$ of a ring, $R, K_{*}(\mathbf{x} ; R)$ denotes the Koszul complex of $R$ with respect to $\mathbf{x}$. If $t$ is a positive integer, $\mathbf{x}^{t}$ denotes the sequence $x_{1}^{t}, \ldots, x_{n}^{t}$.

THEOREM 1.3 (P. RoBerTs). Let $(R, m)$ be a local ring. Then the following two statements are equivalent.

(a) The Canonical Element Conjecture (explained in the appendix) holds for $R$.

(b) For some (equivalently, every) system of parameters $\mathbf{x}=x_{1}, \ldots, x_{n}$ of $R$, for every integer $t \geqslant 1$, and for every free resolution $J_{*}^{t}$ of $R /\left(x^{t}\right)$ and every map of complexes $\lambda_{*}^{t}$ from $K_{*}\left(\mathbf{x}^{t} ; R\right)$ to $J_{*}^{t}$ which lifts the identity map on $R /\left(\mathbf{x}^{t}\right)$, the map $\lambda_{n}^{t}$ splits, i.e. $\lambda_{n}^{t}(1) \notin m J_{n}^{t}$ where $1 \in R \cong K_{n}\left(\mathbf{x}^{t} ; R\right)$.

We record two facts we will need for the proof of Theorem 1.4.

REMARK 1.4. Let $(R, m)$ be an $n$-dimensional local ring of depth $n-1$. Suppose $l\left(H_{m}^{n-1}(R)\right)<\infty$, and let $I=\operatorname{Ann}_{R} H_{m}^{n-1}(R)$. Then

$$
H_{m}^{n-1}(R) \simeq H_{1}\left(K_{*}\left(x_{1}, \ldots, x_{n} ; R\right)\right]
$$

for every system of parameters $x_{1}, \ldots, x_{n}$ of $R$ contained in $I$.

Proof. The assumptions imply $\left\{x_{1}, \ldots, x_{n-1}\right\}$ is an $R$-sequence. Together with the assumption that $\left(x_{1}, \ldots, x_{n}\right) \subset I$, we have

$$
\begin{aligned}
H_{m}^{n-1}(R) & \simeq H_{m}^{0}\left(R /\left(x_{1}, \ldots, x_{n-1}\right)\right) \\
& \simeq\left(x_{1}, \ldots, x_{n-1}: x_{n}\right) /\left(x_{1}, \ldots, x_{n-1}\right) \\
& \simeq H_{1}\left(K_{*}\left(x_{1}, \ldots, x_{n} ; R\right)\right) .
\end{aligned}
$$

REMARK 1.5. Let $(R, m)$ be a local ring of dimension $n$ and let $x_{1}, \ldots, x_{n}$ be a system of parameters of $R$. Let $N=\operatorname{ker}\left(R^{n} \stackrel{\psi}{\rightarrow} R\right)$ where $\psi=\left[x_{1}, \ldots, x_{n}\right]^{t}$. Then the Koszul syzygies, $P_{i j}=-x_{j} e_{i}+x_{i} e_{j}, 1 \leqslant i<j \leqslant n$, where $e_{1}, \ldots, e_{n}$ is the standard basis of $R^{n}$, are part of a minimal system of generators for $N$.

Proof. We first claim that if $y_{1}, y_{2}$ is a system of parameters for a 2-dimensional local ring $(S, q)$, then $y_{1} \notin q\left(y_{1}: y_{2}\right)$. In fact to prove this we may replace $S$ by any local $S$-algebra $T$ for which $y_{1}, y_{2}$ is a system of parameters. We may hence assume that $S$ is a (complete) normal local domain and the claim is now trivial because $\left(y_{1}: y_{2}\right)=\left(y_{1}\right)$.

Suppose $\sum_{i, j} r_{i j} P_{i j} \in m N$ for some $r_{i j} \in R$, where $1 \leqslant i, j \leqslant n$. We need to show $r_{i j} \in m$ for all $1 \leqslant i<j \leqslant n$. Fix $1 \leqslant k<l \leqslant n$. Since

$$
\sum_{i, j} r_{i j} P_{i j}=\sum_{l}\left(\sum_{1 \leqslant i \leqslant l} r_{i l} x_{i}-\sum_{l<j \leqslant n} r_{l j} x_{j}\right) e_{l}
$$

and $\left(s_{1}, \ldots, s_{l}, \ldots, s_{n}\right) \in N$ implies $s_{l} \in\left(x_{1}, \ldots, \hat{x}_{l}, \ldots, x_{n}: x_{l}\right)$, we get

$$
\sum_{1 \leqslant i<l} r_{i l} x_{i}-\sum_{l<j \leqslant n} r_{l j} x_{j} \in m\left(x_{1}, \ldots, \hat{x}_{l}, \ldots, x_{n}: x_{l}\right) \text {. }
$$

Reading (*) modulo $\left(x_{1}, \ldots, \hat{x}_{k}, \ldots, \hat{x}_{l}, \ldots, x_{n}\right) R$, we obtain

$$
\bar{r}_{k l} \bar{x}_{k} \in \bar{m}\left(\bar{x}_{k}: \bar{x}_{l}\right) \text {. }
$$

Hence $r_{k l} \in m$ by our claim. 
Proof OF Theorem 1.1. By Theorem 1.3(b) we need only to consider a system of parameters $x_{1}, \ldots, x_{n}$ lying in $\mathrm{Ann}_{R} H_{m}^{2}(R)$. From Remarks 1.4 and 1.5 we have an exact sequence

$$
0 \rightarrow \operatorname{Ker} \varphi \rightarrow R^{\prime} \oplus R^{3} \stackrel{\varphi}{\rightarrow} R^{3} \stackrel{\psi_{1}}{\rightarrow} R \rightarrow R /\left(x_{1}, x_{2}, x_{3}\right) \rightarrow 0,
$$

where $l$ is the least number of generators of $H_{m}^{2}(R), \psi_{1}=\left[x_{1}, x_{2}, x_{3}\right]^{t}$,

$$
\psi_{2}=\left[\begin{array}{ccc}
-x_{2} & x_{1} & 0 \\
-x_{3} & 0 & x_{1} \\
0 & -x_{2} & x_{2}
\end{array}\right],
$$

and

$$
\varphi=\left[\begin{array}{c}
M \\
-- \\
\psi_{2}
\end{array}\right],
$$

where $M$ is an $l \times 3$ matrix. (Matrices here act on the right.)

Since $H_{1}\left(K_{*}\left(x_{1}, x_{2}, x_{3} ; R\right)\right)$ may be identified with the quotient of the row space of $\varphi$ by the span of the rows of $\psi_{2}$, Remark 1.5 implies that the images of the rows of $M$ minimally generate $H_{1}\left(K_{*}\left(x_{1}, x_{2}, x_{3} ; R\right)\right) \simeq H_{m}^{2}(R)$. Since $H_{m}^{2}(R)$ is assumed to be a direct sum of cyclic modules, we may assume, after elementary row operations on $M$, that $H_{m}^{2}(R)$ is isomorphic to the direct sum of cyclic modules generated by the images of the rows of $M$ in $H_{1}\left(K_{*}\left(x_{1}, x_{2}, x_{3} ; R\right)\right)$. This implies that $\operatorname{Ker} \varphi$ is generated by elements $\left(a_{1}, \ldots, a_{l}, b, c, d\right)$ such that at most one of the $a_{i}$ 's is nonzero.

We denote the standard basis for the copy of $R^{l}$ by $e_{1}, \ldots, e_{l}$. Set $x=0 \oplus$ $\left(x_{3},-x_{2}, x_{1}\right) \in \operatorname{Ker} \varphi$. To complete a proof, we need to show that $x \notin m(\operatorname{Ker} \varphi)$. Suppose the contrary and let $V_{i j}=a_{i j} e_{i} \oplus\left(b_{i j}, c_{i j}, d_{i j}\right), 1 \leqslant i \leqslant l, 1 \leqslant j \leqslant k_{i}$, be elements in $\operatorname{Ker} \varphi$ such that $x=\sum_{i} \sum_{j} r_{i j} V_{i j}$ with $r_{i j} \in m$. Since the component of $x$ in $R^{\prime}$ is $\sum_{i}\left(\sum_{j} r_{i j} a_{i j}\right) e_{i}, \sum_{j} r_{i j} a_{i j}=0$ for $1 \leqslant i \leqslant l$. Since

$$
0 \rightarrow R \stackrel{\left[x_{3}-x_{2} x_{1}\right]}{\rightarrow} R^{3} \stackrel{\psi_{2}}{\rightarrow} R^{3}
$$

is exact (depth $R=2$ ), for each $1 \leqslant i \leqslant l, \sum_{j} r_{i j} V_{i j}=s_{i} x$ for some $s_{i} \in R$. Now $x=\sum_{i} s_{i} x=\left(\sum_{i} s_{i}\right) x$ so that some $s_{i}$, say $s_{l}$, is a unit in $R$. We can then write

$$
x=\sum_{j}\left(r_{l j} / s_{l}\right) V_{j} \quad \text { with }\left(r_{l j} / s_{l}\right) \in m .
$$

Let $N=\operatorname{Ker} \alpha$, where $\alpha: R^{4} \rightarrow R^{3}$ has matrix

$$
\left[\begin{array}{ccc}
z_{1} & z_{2} & z_{3} \\
\hdashline & \psi_{2}
\end{array}\right]
$$

and $\left[\begin{array}{lll}z_{1} & z_{2} & z_{3}\end{array}\right]$ is the $l$ th row of $M$. Let $x^{\prime}=\left(0, x_{3},-x_{2}, x_{1}\right)$ and $V_{l j}^{\prime}=$ $\left(a_{l j}, b_{l j}, c_{l j}, d_{l j}\right), 1 \leqslant j \leqslant k_{l}$. (These are the projections of $x$ and $V_{l j}$ onto the last four coordinates.) Then $x^{\prime} \in N$ and $V_{l j}^{\prime} \in N$ for all $1 \leqslant j \leqslant k_{l}$. From (*), we get $x^{\prime} \in m N$ which is a contradiction by the Eisenbud-Evans-Bruns generalized principal ideal theorem (see [2]) because $\left\{f\left(x^{\prime}\right): f \in \operatorname{Hom}_{R}(N, R)\right\}$ contains the ideal 
$\left(x_{1}, x_{2}, x_{3}\right)$ of height 3 and rank $N \leqslant 2$ because the exactness of ( $\dagger$ ) implies $\operatorname{rank}\left(\operatorname{Im}\left(\psi_{2}\right)\right)=2$ so that $\operatorname{rank}(\operatorname{Im}(\alpha)) \geqslant 2$. (Recall that the rank of an $R$-module $M$ is $\max \left\{\operatorname{dim}_{k(p)}\left(M_{p} / p M_{p}\right): p\right.$ is a minimal prime of $\left.R\right\}, k(p)=R_{p} / p R_{p}$.)

PROOF OF THEOREM 1.2. By Theorem 1.1 and [6, (2.4) Corollary], it will suffice to show that there is a 3-dimensional local $R$-algebra $(S, q)$ of depth 2 such that $H_{q}^{2}(S)$ is cyclic and such that a system of parameters of $R$ is mapped onto a system of parameters for $S$ under the structural homomorphism. We may therefore assume $R$ is complete. Let $q_{1} \cap \cdots \cap q_{l}=0$ be a primary decomposition of zero in $R$. Let $q=\bigcap\left\{q_{i}: \operatorname{dim}\left(R / q_{i}\right)>1\right\}$ and $q^{\prime}=\bigcap\left\{q_{j}: \operatorname{dim}\left(R / q_{j}\right) \leqslant 1\right\}$. Since $q^{\prime} \subset \operatorname{Ann}_{R} q$,

$$
\operatorname{dim} q=\operatorname{dim}\left(R / \operatorname{Ann}_{R} q\right) \leqslant \operatorname{dim}\left(R / q^{\prime}\right) \leqslant 1 .
$$

From the long exact sequence of local cohomology induced by $0 \rightarrow q \rightarrow R \rightarrow R / q$ $\rightarrow 0$, we get $H_{m}^{2}(R / q) \cong H_{m}^{2}(R)$ since $H_{m}^{2}(q)=H_{m}^{3}(q)=0$ as $\operatorname{dim}(q) \leqslant 1$. We may thus assume that $(R, m)$ is a 3 -dimensional complete local ring of depth $\geqslant 1$ such that $\operatorname{dim}(R / p)>1$ for all $p \in \operatorname{Ass}(R)$. Let

$$
\begin{aligned}
& S=\underset{\vec{n}}{\lim _{n}} \operatorname{Hom}\left(m^{n}, R\right) \\
& \cong\{x / y \in \text { total quotient ring of } R: x, y \in R, \\
&\left.y \text { not a zero divisor, } x m^{n} \subset y R \text { for some } n\right\} .
\end{aligned}
$$

The short exact sequence $0 \rightarrow m^{n} \rightarrow R \rightarrow R / m^{n} \rightarrow 0$ induces the exact sequence

$$
0 \rightarrow \operatorname{Hom}\left(R / m^{n}, R\right) \rightarrow R \rightarrow \operatorname{Hom}\left(m^{n}, R\right) \rightarrow \operatorname{Ext}^{1}\left(R / m^{n}, R\right) \rightarrow 0 .
$$

Taking the direct limit, we get an exact sequence

$$
0 \rightarrow R \rightarrow S \rightarrow H_{m}^{1}(R) \rightarrow 0 .
$$

By [1, (3.7) Corollary], $H_{m}^{1}(R)$ has finite length. Now the long exact sequence of local cohomology induced by (\#) gives $H_{m}^{0}(S)=H_{m}^{1}(S)=0$ and $H_{m}^{2}(S) \cong H_{m}^{2}(R)$. Hence $H_{m}^{2}(S)$ is cyclic as an $S$-module. Localizing $S$ at a maximal ideal $q$ of ht 3 , we get the desired local $R$-algebra because $H_{q S_{q}}^{i}\left(S_{q}\right) \cong H_{m}^{i}(S) \otimes_{S} S_{q}$.

APPENDIX. We give the proof due to the referee of Theorem 1.3. First we review the statement of the Canonical Element Conjecture. Let $(R, m)$ be a local ring of dimension $n$. Let $F_{*}$ be a free resolution of $R / m$. Then the Canonical Element Conjecture asserts that, for every system of parameters $\mathbf{x}=x_{1}, \ldots, x_{n}$ for $R$ and for every map of complexes $\psi_{*}$ from $K_{*}(\mathbf{x} ; R)$ to $F_{*}, \psi_{n}(1) \neq 0$, or equivalently, the element $\eta_{\mathbf{x}}$ of $\operatorname{Tor}_{n}^{R}(R / m, R /(\mathbf{x}))$ induced by $\psi_{n}(1) \otimes 1_{R /(\mathbf{x})} \in F_{n} \otimes R /(\mathbf{x})$ is not zero.

We can state (a) of Theorem 1.3 as $\left(\mathrm{a}^{\prime}\right)$ : For some (equivalently, every by Remarks (2.2) of [6]) system of parameters $\mathbf{x}=x_{1}, \ldots, x_{n}, \eta_{x} t \neq 0$ for all $t \geqslant 1$.

On the other hand, we denote by $\rho_{\mathrm{x}}$ the element of $\operatorname{Tor}_{n}^{R}(R / m, R /(\mathbf{x}))$ induced by $1_{R / m} \otimes \lambda_{n}(1) \in R / m \otimes J_{n}$, where $\lambda_{*}$ and $J_{*}$ are as in Theorem 1.3. Then we can restate (b) of Theorem 1.3 as

(b') For some (equivalently, every) system of parameters $\mathbf{x}=x_{1}, \ldots, x_{n}$ for $R$, $\rho_{\mathrm{x}} t \neq 0$ for all $t \geqslant 1$. Thus to prove Theorem 1.3 it suffices to show that for every system of parameters $\mathrm{x}$ of $R, \eta_{\mathrm{x}}$ and $\rho_{\mathrm{x}}$ represent the same element of $\operatorname{Tor}_{n}^{R}(R / m, R /(\mathbf{x}))$, which we think of as $(n-1)$ th homology of the total complex 
of $\left(F_{*} \rightarrow R / m \rightarrow 0\right) \otimes\left(J_{*} \rightarrow R /(\mathbf{x}) \rightarrow 0\right)$ where $R / m$ and $R /(\mathbf{x})$ are at $(-1)$ th position. Let $K_{*}=K_{*}(\mathbf{x} ; R), \psi_{*}=\psi_{*, \mathbf{x}}$, and $\lambda_{*}=\lambda_{*, \mathbf{x}}$. We are free to choose $\psi_{*}$ so that it factors $K_{*} \rightarrow J_{*} \rightarrow F_{*}$, with the first map equal to $\lambda_{*}$. We thus are reduced to proving that, if $\overline{1}$ denotes the identity in $R /(\mathbf{x})$, then the elements $\lambda_{n}(1) \otimes \overline{1} \in J_{n} \otimes R /(\mathbf{x})$ and $\overline{1} \otimes \lambda_{n}(1) \in R /(\mathbf{x}) \otimes J_{n}$ represent the same element in $\operatorname{Tor}_{n}^{R}(R /(\mathbf{x}), R /(\mathbf{x}))$, for these map to $\eta_{\mathrm{x}}$ and $\rho_{\mathrm{x}}$ respectively under the natural map to $\operatorname{Tor}_{n}^{R}(R / m, R /(\mathbf{x}))$. Let $A=\mathbf{Z}\left[X_{1}, \ldots, X_{n}\right]$, where $\mathbf{Z}$ is the integers, and let $\mathbf{Z}$ also denote the $A$-module $A /(\mathbf{x})$. It is easy to see that if we construct the corresponding two elements in $\operatorname{Tor}_{n}^{A}(\mathbf{Z}, \mathbf{Z}) \cong \mathbf{Z}$ they are equal (to 1$)$ and the general case follows at once from this: map $A \rightarrow R$ by sending $X_{i}$ to $x_{i}$ for each $i$, and use the fact that the elements we want are the images of the two in $\operatorname{Tor}_{n}^{A}(\mathbf{Z}, \mathbf{Z})$ under the natural map

$$
\operatorname{Tor}_{n}^{A}(\mathbf{Z}, \mathbf{Z}) \rightarrow \operatorname{Tor}_{n}^{R}(\mathbf{Z} \otimes R, \mathbf{Z} \otimes R)
$$

\section{REFERENCES}

1. M. Brodmann, Finiteness of ideal transforms, J. Algebra 63 (1980), 162-185.

2. W. Bruns, The Eisenbud-Evans principal ideal theorem and determinantal ideals, Proc. Amer. Math. Soc. 83 (1981), 19-24.

3. D. Eisenbud and E. G. Evans, Jr., A generalized principal ideal theorem, Nagoya Math. J. 62 (1976), 41-53.

4. A. Grothendieck (notes by R. Hartshorne), Local cohomology, Lecture Notes in Math., vol. 41, Springer-Verlag, 1967.

5. M. Hochster, Topics in the homological theory of modules over commutative rings, CBMS Regional Conf. Ser. in Math., no. 24, Amer. Math. Soc., Providence, R. I., 1975.

6. Canonical elements in local cohomology modules and the direct summand conjecture, J. Algebra 84 (1983), 503-553.

7. J. Koh, Degree $p$ extensions of an unramified regular local ring of mixed characteristic p, J. Algebra (to appear).

8. P. Roberts, The equivalence of two forms of the Canonical Element Conjecture, preprint.

Department of Mathematics, Purdue University, West Lafayette, Indiana 47907 